\section{Avaliação Auditiva (audiometria tonal e emissões otoacústica evocadas - produtos de distorção) em pacientes portadores de Lúpus Eritematoso Sistêmico e Artrite Reumatóide}

\author{
Súnia Ribeiro' ${ }^{1}$, Chiara L. de O. Moraes ${ }^{1}$, \\ Gabriele Righetti Neto ${ }^{1}$, \\ José Alexandre Médicis da Silveira ${ }^{2}$, \\ Fabiana Gonçalez ${ }^{3}$, Sandra Murad Quintero ${ }^{4}$
}

Resumo

$\mathbf{O}$

bjetivo: Avaliar o comportamento clínico e auditivo dos pacientes com doença auto-imune (DAI), em particular Artrite Reumatóide (AR) e Lúpus Eritematoso Sistêmico (LES) e comparar os achados audiométricos e às emissões otoacústicas (EOAs) - produtos de distorção nestes pacientes. Casuística e Método: Foram selecionados 40 pacientes com DAI (AR e LES), sendo 27 portadores de AR e 13 de LES, 95\% do sexo feminino, mediana 56 anos no grupo de AR e 29 anos no grupo de LES. A avaliação auditiva foi realizada através de anamnese dirigida, exame otorrinolaringológico, audiometria tonal e emissões otoacústicas evocadas (produtos de distorção). Resultados: Foram estudadas 80 orelhas sendo observada alteração à audiometria tonal em 19 (19/54 - 35\%) no grupo de AR e, em apenas quatro (4/26-15,4\%) no grupo de LES. Em relação às Otoemissões, foi detectada alteração em 37 orelhas $(37 / 54-68,5 \%)$ do grupo de AR e em oito $(8 / 26-30,7 \%)$ no grupo de LES. Das 80 orelhas com DAI, $23(28,8 \%)$ apresentaram audiometria alterada e $45(56,3 \%)$ alterações às otoemissões. Conclusão: Os autores sugerem que as emissões otoacústicas (EOAs) produtos de distorção sejam mais sensíveis que a audiometria tonal na detecção de alterações cocleares nas doenças auto-imune, fazendose necessário um seguimento a longo prazo para que se confirme o valor preditivo das EOAs no prognóstico audiológico dos pacientes com DAI.
Auditory Evaluation (tone audiometry and evoked otoacustic emissions - distortion product) in patients with Systemic Lupus Erytematosus and Rheumatoid Arthritis

Palavras-chave: audiometria tonal, emissões otoacústicas, lupus eritematoso sistêmico, artrite reumatóide.

Key words: tonal audiometry, otoacustic emissions, systemic lupus erytematosus, rheumatoid arthritis.

Médico Residente do Serviço de Otorrinolaringologia do Hospital Santa Marcelina.

${ }^{2}$ Chefe do Serviço de Otorrinolaringologia do Hospital Santa Marcelina, Diretor da Clínica Otorhinus e Assistente-Doutor da Clínica ORL do Hospital das Clínicas da Faculdade de Medicina de São Paulo.

${ }_{3}^{3}$ Médica preceptora dos Residentes da Clínica Otorhinus e do Hospital Santa Marcelina. ${ }^{4}$ Fonoaudióloga da Clínica Otorhinus.

Trabalho realizado no Hospital Santa Marcelina em conjunto com a Clínica Otorhinus (Centro de Estudos Alexandre Médicis da Silveira), São Paulo/SP. Endereço para correspondência: Súnia Ribeiro - Rua Luís Góes,1328/6 - São Paulo/SP - CEP 04043-150 - Tel:(0xx11) 5581.7464

Artigo recebido em 3 de dezembro de 2001. Artigo aceito em 7 de fevereiro de 2002. 


\section{INTRODUÇÃO}

O estudo das doenças imunomediadas do ouvido interno é relativamente novo. Teve início há cerca de quatro décadas atrás, primeiro na Alemanha e posteriormente no Japão, com estudos de Lehnhart ${ }^{1}$ em 1958, sobre anticorpos anticocleares, e Kikuchi ${ }^{2}$, sobre a chamada otite do simpático, em 1959. Beichert ${ }^{3}$, em 1961, observou reações auto-imunes em cortes histológicos da cóclea.

A partir da década de 70 iniciou-se um período de melhor conhecimento das lesões do ouvido interno. A perda auditiva neurossensorial, que até então era vista com reservas por apresentar tratamento pouco recompensador, passou a ser melhor compreendida graças à realização de inúmeros estudos sobre os mecanismos etiopatogênicos envolvidos ${ }^{4,5,6}$.

McCabe $^{7}$, em 1979, relatou 17 casos de perda auditiva neurossensorial que apresentaram boa resposta ao tratamento com corticosteróides. Neste mesmo estudo o autor verificou ainda a presença de respostas alteradas ao teste de migração linfocitária, pressupondo a participação de mecanismo imunológico e, pela primeira vez, o diagnóstico de disacusia neurossensorial auto-imune foi apresentado na literatura.

O conhecimento das doenças auto-imunes e sua correlação com o quadro de disacusia neurossensorial (DNS) contribuiu de maneira importante na desmistificação deste tipo de perda auditiva permitindo a instituição de tratamento específico.

As doenças auto-imunes (DAI) podem ser classificadas em doenças órgão-específicas (primárias) anticorpos dirigidos aos antígenos de apenas um órgão do corpo, como a tireoidite de Hashimoto, e doenças não órgão-específicas (secundárias) - anticorpos dirigidos aos antígenos de diversos tecidos levando a doença sistêmica, como é o caso do Lúpus Eritematoso Sistêmico (LES).

O ouvido interno pode se situar dentro destes grupos como doença órgão-específica ou, mais comumente, como órgão comprometido dentro da forma sistêmica, podendo a lesão auditiva ser, neste caso, o primeiro sintoma. $\mathrm{O}$ índice de comprometimento do ouvido interno das disacusias autoimunes sistêmicas é variável. Doig e cols. ${ }^{6}$ observaram DNS em quase 50\% dos pacientes com Artrite Reumatóide (AR). Outros autores correlacionaram mecanismos imunológicos na patogênese de várias formas de comprometimento auditivo, como perda auditiva flutuante, disacusia neurossensorial rapidamente progressiva, surdez súbita, e até alguns quadros do tipo Ménière ${ }^{8,9}$.

A DNS envolvendo mecanismo imunológico é mais comumente encontrada em pessoas do sexo feminino, entre a $3^{a}$ e a $5^{a}$ décadas de vida, apresentando instalação insidiosa e podendo progredir para perda auditiva moderada a severa em poucos meses.

$\mathrm{Na}$ série de $\mathrm{McCabe}^{10}$, a idade dos pacientes variou de 19 a 42 anos, sem diferença quanto ao sexo. Hughes et $a^{11}$ relataram que a perda auditiva pode começar em qualquer idade, sendo mais comum em mulheres de meia idade.

As curvas audiométricas não apresentam característica padrão na DNS auto-imune, podendo ser ascendentes ou descendentes. A discriminação vocal pode estar ou não alterada, independente da curva tonal.

O registro das emissões otoacústicas evocadas (EOAs) é o mais novo método para detecção de alterações auditivas de origem coclear. As EOAs foram primeiramente observadas pelo inglês David Kemp ${ }^{12}$ em 1978 que as definiu como liberação de energia sonora originada na cóclea que se propaga na orelha média até alcançar o conduto auditivo externo.

As EOAs podem ser classificadas em duas categorias: as espontâneas e as evocadas ${ }^{13}$. As evocadas são divididas em 3 tipos, de acordo com a natureza do estímulo utilizado: transiente, estímulo-freqüência e produtos de distorção. Os produtos de distorção, utilizados em nosso trabalho, são obtidos por estímulo sonoro tipo click, composto por dois tons puros de freqüências diferentes (f1 e f2). A resposta é caracterizada pela ocorrência de um terceiro tom cuja freqüência é um produto de distorção tipicamente 2f1-f2, sendo que a relação f1/f2 é aproximadamente 1,2 $2^{14,15}$.

O produto de distorção é um tipo de emissão que surge quase que instantaneamente com estímulo acústico, e pode ser gerado em qualquer freqüência, por meio de estímulos selecionados, avaliando a atividade da cóclea em freqüências específicas. Devido à sua maior semelhança com a curva audiométrica e a possibilidade da avaliação da função das células ciliadas em diferentes pontos da cóclea, os produtos de distorção proporcionam mais ampla aplicação clínica quando comparados às emissões evocadas transitórias. As emissões otoacústicas evocadas por produto de distorção podem ser obtidas nas freqüências entre 0,75 e $8 \mathrm{KHz}$, e nos indivíduos com limiares de até aproximadamente $50 \mathrm{~dB} \mathrm{NA}^{16}$.

Os objetivos deste trabalho são avaliar o comportamento clínico e auditivo dos pacientes com DAI, em particular portadores de AR e LES e comparar os sintomas com os achados audiométricos e das emissões otoacústicas (produtos de distorção) nestes pacientes.

\section{CASUÍSTICA E MÉTODO}

Foram selecionados 40 pacientes com quadro clínico estabelecido de doença auto-imune (Artrite Reumatóide e Lúpus Eritematoso Sistêmico) acompanhados pelo ambulatório do Departamento de Reumatologia do Hospital Santa Marcelina, São Paulo, no período de março de 2000 a março de 2001.

Os critérios de exclusão para o grupo estudado incluíram: contato com ruídos de alta intensidade, doenças da orelha média, história familiar de perda auditiva, utilização 
prévia de aminoglicosídeos e/ou diuréticos de alça e sífilis.

Dentre os 40 pacientes selecionados, 38 (95\%) eram do sexo feminino e dois (5\%) do sexo masculino, sendo 27 (67,5\%) portadores de AR e 13 (32,5\%) de LES.

A idade variou de 16 a 71 anos (mediana de 56 anos) no grupo de AR e de 16 a 40 anos (mediana de 29 anos) no grupo de LES.

Os pacientes foram questionados com relação à presença de zumbido, vertigem/tontura e plenitude auricular e foram submetidos a exame otorrinolaringológico e otoneurológico, incluindo os testes de Fukuda, Romberg, Romberg sensibilizado, presença de nistagmo espontâneo e semi-espontâneo, testes cerebelares e avaliação dos pares cranianos. Foram ainda submetidos a audiometria e impedanciometria, emissões otoacústicas evocadas por produtos de distorção e sorologia para lues. Os exames auditivos foram realizados na Clínica Otorhinus, São Paulo. A audiometria tonal foi realizada em cabine acústica, utilizando-se aparelho MAICO-MA 41 e imitância acústica com impedanciômetro Rexton Impedance Audiometer 128. Para registro das Emissões Otoacústicas Evocadas (Produtos de Distorção) foi utilizado o programa Scout Sport (Distortion Product Otoacoustic Emissions Measurement System - program version 1.54) da Biologin Systems Corporation avaliando-se as freqüências de 0,75 a $8 \mathrm{KHz}$.

Foram estudados em separado os grupos de artrite reumatóide e lúpus eritematoso sistêmico correlacionando a presença de sintomas com os achados audiométricos e às emissões otoacústicas por produtos de distorção utilizando-se o teste de X2 para $\mathrm{p}<0,005$. A mesma análise foi realizada avaliando o grupo como um todo.

\section{RESULTADOS}

Dentre as 80 orelhas estudadas a queixa mais freqüente foi o zumbido encontrado em 30 orelhas $(37,5 \%)$, seguido de plenitude auricular em 9 orelhas (11,25\%). Dentre as orelhas com zumbido, 20 (66,7\%) eram do grupo de AR e 10 (33,3\%) do grupo de LES.

Vertigem/tontura foi relatada em 14 pacientes (35\%), sendo nove $(64,3 \%)$ do grupo de AR e cinco $(35,7 \%)$ do grupo de LES.

Dentre os 40 pacientes estudados, não foram observadas alterações ao exame de pares cranianos e testes de equilíbrio, além do exame cerebelar e pesquisa de nistagmo.

Devido à falta de consenso para se classificar perda auditiva utilizando-se apenas freqüências isoladas, os autores optaram pela análise do limiar tonal puro de cada freqüência isoladamente, considerando a audição normal de -10 a 25 dBNA.

Foi observada alteração à audiometria tonal em 19 orelhas (19/54 - 35\%) no grupo de AR e em apenas quatro
(4/26 - 15,4\%) no grupo de LES. Das 80 orelhas com DAI (AR e LES) 23 (28,8\%) apresentaram audiometria alterada. (Gráfico 1).

Dentre as 80 orelhas estudadas, a perda neurossensorial nas freqüências agudas $(4,6 \mathrm{e} / \mathrm{ou} 8 \mathrm{kHz})$ foi mais prevalente (22/23-95,6\%) à audiometria tonal, o mesmo ocorrendo com as otoemissões 37/45 (82,2\%).

Em relação às otoemissões, 37 orelhas (68,5\%) do grupo de AR apresentaram alterações, sendo que dentre estas, 18 (48,6\%) tinham audiometria normal. No grupo de LES, em oito orelhas $(30,7 \%)$ foram detectadas anormalidades, e destas, quatro (50\%) apresentavam-se audiometricamente normais. Das 80 orelhas com DAI (AR e LES), 45 (56,3\%) apresentaram otoemissões alteradas (Gráfico 1).

No grupo de artrite reumatóide houve significância estatística na correlação dos achados de audiometria e EOA, assim como na presença de sintomas e alterações audiométricas. Não houve correlação significativa entre os sintomas e as otoemissões, tampouco quando comparados sintomas e achados audiométricos e às otoemissões (Tabela 1).

No grupo de lúpus eritematoso sistêmico houve significância estatística na correlação dos achados de audiometria e presença de sintomas, assim como na presença de sintomas e alterações às otoemissões. Não houve correlação significativa entre os achados de audiometria e otoemissões, tampouco quando comparados sintomas e achados audiométricos e às otoemissões (Tabela 2). Estudados os dois grupos em conjunto houve significância estatística na correlação dos achados de audiometria e otoemissões, assim como na presença de sintomas e alterações audiométricas. Não houve correlação significativa entre a presença de sintomas e alterações às otoemissões, tampouco quando comparados sintomas e achados audiométricos e às otoemissões (Tabela 3).

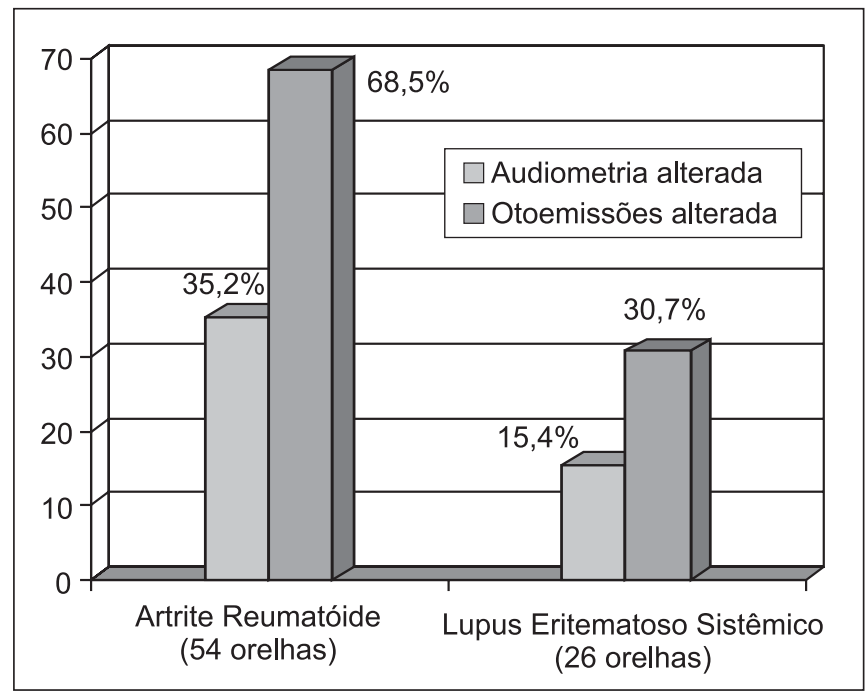

Gráfico 1. Distribuição dos Achados Audiológicos ( $n=80$ orelhas) 
Tabela 1. Sintomas $x$ Achados Audiológicos: Artrite Reumatóide ( $n=54$ orelhas)

\begin{tabular}{|c|c|c|c|c|c|c|c|}
\hline \multicolumn{2}{|c|}{$\begin{array}{c}\text { Audio nl } \\
\mathrm{N}=35 / 54\end{array}$} & \multicolumn{2}{|c|}{$\begin{array}{l}\text { Audio alt } \\
\mathrm{N}=19 / 54\end{array}$} & \multicolumn{2}{|c|}{$\begin{array}{c}\text { EOAs nl } \\
\mathrm{N}=17 / 54\end{array}$} & \multicolumn{2}{|c|}{$\begin{array}{l}\text { EOAs alt } \\
\mathrm{N}=37 / 54\end{array}$} \\
\hline $\mathrm{C} / \sin t$ & $S / \sin t$ & $\mathrm{C} / \sin t$ & $S / \operatorname{sint}$ & $\mathrm{C} / \sin t$ & $S / \sin t$ & $\mathrm{C} / \sin t$ & S/sint \\
\hline $\begin{array}{l}20 / 35 \\
(57 \%) \\
\end{array}$ & $\begin{array}{l}15 / 35 \\
(43 \%)\end{array}$ & $\begin{array}{c}11 / 19 \\
(58 \%)\end{array}$ & $\begin{array}{c}08 / 19 \\
(42 \%)\end{array}$ & $\begin{array}{l}02 / 17 \\
(12 \%)\end{array}$ & $\begin{array}{l}15 / 17 \\
(88 \%)\end{array}$ & $\begin{array}{l}20 / 37 \\
(54 \%)\end{array}$ & $\begin{array}{l}17 / 37 \\
(46 \%)\end{array}$ \\
\hline
\end{tabular}

Análise estatística (AR) $(p<0,005)$

\begin{tabular}{ll}
\hline Audio X EOAs & $\mathrm{P}_{1}=0,000375$ \\
Sintomas X Audio & $\mathrm{P}_{1}=0,001357$ \\
Sintomas X EOAs & $\mathrm{P}_{1}=0,148914$ \\
Sintomas X Audio X EOAs & $\mathrm{P}_{1}=0,108$ \\
\hline
\end{tabular}

Tabela 2. Sintomas $x$ Achados Audiológicos: Lupus Eritematoso Sistêmico ( $n=26$ orelhas)

\begin{tabular}{|c|c|c|c|c|c|c|c|}
\hline \multicolumn{2}{|c|}{$\begin{array}{c}\text { Audio nl } \\
\mathrm{N}=22 / 26\end{array}$} & \multicolumn{2}{|c|}{$\begin{array}{l}\text { Audio alt } \\
\mathrm{N}=04 / 26\end{array}$} & \multicolumn{2}{|c|}{$\begin{array}{c}\text { EOAs nl } \\
\mathrm{N}=18 / 26\end{array}$} & \multicolumn{2}{|c|}{$\begin{array}{l}\text { EOAs alt } \\
N=08 / 26\end{array}$} \\
\hline $\mathrm{C} / \operatorname{sint}$ & S/sint & $\mathrm{C} / \operatorname{sint}$ & $\mathrm{S} / \operatorname{sint}$ & $\mathrm{C} / \operatorname{sint}$ & $\mathrm{S} / \operatorname{sint}$ & $\mathrm{C} / \operatorname{sint}$ & S/sint \\
\hline $\begin{array}{l}12 / 22 \\
(55 \%)\end{array}$ & $\begin{array}{l}10 / 22 \\
(46 \%)\end{array}$ & $\begin{array}{l}03 / 04 \\
(75 \%)\end{array}$ & $\begin{array}{l}01 / 04 \\
(25 \%)\end{array}$ & $\begin{array}{l}10 / 18 \\
(55 \%)\end{array}$ & $\begin{array}{l}08 / 18 \\
(44 \%)\end{array}$ & $\begin{array}{l}04 / 08 \\
(50 \%)\end{array}$ & $\begin{array}{l}04 / 08 \\
(50 \%)\end{array}$ \\
\hline \multicolumn{8}{|c|}{ Análise Estatística (LES)" $(p<0,005)$} \\
\hline \multicolumn{4}{|c|}{$\begin{array}{l}\text { Audio X EOAs } \\
\text { Sintomas X Audio } \\
\text { Sintomas X EOAs } \\
\text { Sintomas X Audio }\end{array}$} & & $\begin{array}{l}P_{1}=0 \\
P_{1}=8, \\
P_{1}=0,0 \\
P_{1}=0,2\end{array}$ & $\begin{array}{l}317 \\
\mathrm{E}-05 \\
4922 \\
4508\end{array}$ & \\
\hline
\end{tabular}

Tabela 3. Sintomas $x$ Achados Audiológicos: AR e LES ( $n=80$ orelhas)

\begin{tabular}{|c|c|c|c|c|c|c|c|}
\hline \multicolumn{2}{|c|}{$\begin{array}{l}\text { Audio nl } \\
\mathrm{N}=57 / 80\end{array}$} & \multicolumn{2}{|c|}{$\begin{array}{l}\text { Audio alt } \\
\mathrm{N}=23 / 80\end{array}$} & \multicolumn{2}{|c|}{$\begin{array}{c}\text { EOAs nl } \\
\mathrm{N}=35 / 80\end{array}$} & \multicolumn{2}{|c|}{$\begin{array}{l}\text { EOAs alt } \\
\mathrm{N}=45 / 80\end{array}$} \\
\hline $\mathrm{C} / \sin t$ & $S / \operatorname{sint}$ & $\mathrm{C} / \sin t$ & $S / \operatorname{sint}$ & $\mathrm{C} / \operatorname{sint}$ & $S / \operatorname{sint}$ & $\mathrm{C} / \operatorname{sint}$ & $S / \operatorname{sint}$ \\
\hline $\begin{array}{l}32 / 57 \\
(56 \%)\end{array}$ & $\begin{array}{l}35 / 57 \\
(61 \%)\end{array}$ & $\begin{array}{l}14 / 23 \\
(61 \%)\end{array}$ & $\begin{array}{l}09 / 23 \\
(39 \%)\end{array}$ & $\begin{array}{l}12 / 35 \\
(34 \%)\end{array}$ & $\begin{array}{l}23 / 35 \\
(66 \%)\end{array}$ & $\begin{array}{l}24 / 45 \\
(53 \%)\end{array}$ & $\begin{array}{l}21 / 45 \\
(47 \%)\end{array}$ \\
\hline
\end{tabular}

Análise Estatística (LES) $(p<0,005)$

\begin{tabular}{ll}
\hline Audio X EOAs & $\mathrm{P}_{1}=0,11317$ \\
Sintomas X Audio & $\mathrm{P}_{1}=8,63 \mathrm{E}-05$ \\
Sintomas X EOAs & $\mathrm{P}_{1}=0,004922$ \\
Sintomas X Audio X EOAs & $\mathrm{P}_{1}=0,294508$ \\
\hline
\end{tabular}

\section{DISCUSSÃO}

Desde as publicações de McCabe ${ }^{7,10}$, sugerindo pela primeira vez a relação clínica de DNS com DAI, pôde-se observar, a partir de 1979, um maior número de publicações aventando ser a causa auto-imune a principal hipótese diagnóstica para muitos casos de DNS sem causa aparente $\mathrm{e}^{9,11,17}$.

A peça fundamental no diagnóstico de DNS autoimune é a história clínica, visto que ainda não existem testes laboratoriais definitivos ${ }^{16}$. A prova histopatológica ainda não é possível, uma vez que a orelha interna é inacessível para biópsia tecidual. Bowman e Nelson ${ }^{18}$ mostraram que a associação entre DNS auto-imune e o HLA-CW7 poderá ser um marcador diagnóstico que facilitará a seleção dos pacientes para tratamento.

A DNS pode se manifestar inicialmente como um sintoma isolado, porém é mais comumente parte de uma doença sistêmica imunomediada ${ }^{9,11,19}$.

O presente trabalho, em conformidade com o de Hughes, ${ }^{10}$ também mostrou que DNS envolvendo mecanismo imunológico pode se iniciar em qualquer idade, sendo mais comum em mulheres.

Sâmara et $\mathrm{al}^{20}$ estudaram 18 pacientes com AR graus I a IV quanto aos resultados da audiometria tonal e impedanciometria. Concluíram que, embora não se tenha conseguido estabelecer qualquer relação entre intensidade das manifestações articulares e as disfunções da orelha interna, a ocorrência de evidências de disacusia latente (audiometria normal e recrutamento presente) e neurossensorial (perda auditiva para agudos e recrutamento presente) em 66,6\% dos pacientes, não vinculados ao grupo etário ou outras condições mórbidas, parece sugerir uma forma de agressão reumatóide às estruturas cocleares. No presente trabalho, dentre as 54 orelhas do grupo de AR, 19 orelhas (35\%) mostraram alteração à audiometria tonal e 37 orelhas $(68,5 \%)$ apresentaram alterações às otoemissões, sendo que dentre estas, 18 (48,6\%) tinham audiometria normal. Ainda no presente trabalho houve significância estatística na correlação dos achados de audiometria e EOA no grupo de artrite reumatóide, assim como na presença de sintomas e alterações audiométricas. Não houve correlação significativa entre os sintomas e as otoemissões, tampouco quando comparados sintomas e achados audiométricos e às otoemissões neste grupo.

Atra et $\mathrm{al}^{21}$ realizaram audiometria tonal, vocal e impedanciometria em 30 pacientes com LES e compararam com grupo controle. Os resultados foram normais nos dois grupos, e concluíram que o LES e o tratamento medicamentoso desta doença de base não produziram alterações na audição. Em discordância com estes achados, nossos resultados mostraram que os pacientes com LES apresentaram audiometria alterada em quatro orelhas (4/26 $15,4 \%)$ sendo que em oito $(30,70 \%)$ houve alteração nas emissões otoacústicas (produtos de distorção).

Das 80 orelhas com DAI (AR e LES) $23(28,8 \%)$ apresentaram audiometria alterada e $45(56,3 \%)$ alterações às otoemissões. A perda neurossensorial nas freqüências agudas (4,6 e/ou $8 \mathrm{KHz})$ foi mais prevalente $(22 / 23-95,6 \%)$ à audiometria tonal, o mesmo ocorrendo com as otoemissões $(37 / 45-82,2 \%)$.

Quando foram comparados nos diferentes grupos a presença de sintomas com os achados audiométricos e as alterações às emissões otoacústicas, os autores observaram que houve significância estatística na correlação dos 
achados de audiometria e EOA, assim como na presença de sintomas e alterações audiométricas no grupo de artrite reumatóide. Não houve correlação significativa entre os sintomas e as otoemissões, tampouco quando comparados sintomas e achados audiométricos e às otoemissões. Já no grupo de lúpus eritematoso sistêmico houve significância estatística na correlação dos achados de audiometria e presença de sintomas, assim como na presença de sintomas e alterações às otoemissões. Não houve correlação significativa entre os achados de audiometria e otoemissões, tampouco quando comparados sintomas e achados audiométricos e às otoemissões. Estudados os dois grupos em conjunto houve significância estatística na correlação dos achados de audiometria e otoemissões, assim como na presença de sintomas e alterações audiométricas. Não houve correlação significativa entre a presença de sintomas e alterações às otoemissões, tampouco quando comparados sintomas e achados audiométricos e às otoemissões.

Uma possível explicação para a não-correlação estatisticamente significativa da presença de sintomas com as alterações audiométricas e às otoemissões, seja nos grupos individuais ou no estudo das 80 orelhas como um todo, seria a ocorrência de alterações bioquímicas ou fisiológicas sem lesão anatômica? .

Segundo os resultados expostos acima, os autores sugerem a possibilidade das emissões otoacústicas serem um exame mais sensível que a audiometria tonal na detecção de alterações cocleares nas doenças autoimunes. Para tal afirmativa, um grupo maior de pacientes deve ser estudado e aqueles com alteração às emissões otoacústicas devem ser seguidos quanto a evolução para disacusia neurossensorial, confirmando o valor preditivo deste exame no prognóstico audiológico dos pacientes com DAI.

\section{CONCLUSÃO}

Com o presente trabalho, os autores objetivaram estudar os pacientes com doença auto-imune (especificamente LES e AR) e comparar os sintomas (vertigem/tontura, plenitude auricular e zumbido) com os achados audiométricos e às otoemissões. Tendo sido as alterações audiométricas e às otoemissões mais encontradas nas freqüências agudas $(4,6,8 \mathrm{KHz})$ os autores enfatizam a importância das emissões otoacústicas na avaliação dos pacientes com DAI.

Os resultados deste estudo sugerem que as emissões otoacústicas (produtos de distorção) sejam mais sensíveis que a audiometria tonal na detecção de alterações cocleares nas doenças auto-imunes, devendo ser dado seguimento ao estudo para que se confirme o valor preditivo das otoemissões no prognóstico audiológico dos pacientes com DAI.
Os autores enaltecem a importância em se orientar os portadores de DAI quanto à possibilidade de comprometimento da orelha interna durante a evolução da doença de base, e propor que a avaliação audiológica seja realizada periodicamente neste grupo.

\section{REFERÊNCIAS BIBLIOGRÁFICAS}

1. Lehnhart E. Plötzliche Hörstörungen auf briden Seiten gleichzeitig oder nacheinander aujgetreren. Z Laryngol Rhinol Oto 1958;37:117 .

2. Kikuchi M. On the "sympathetic otitis". Zibi Rinsyo Kyoto 1959;52:600.

3. Beichert P. Zur Frage der Empfindungs Schwerhorigkeit und Autoallergie. Z Laryngol Rhinol Otol 1961;40:837.

4. Arnold W, Pjaltz R, Altermatt HF. Evidence of serum antibodies against inner ear tissues in the blood of patients with certain sensorineural hearing disorders. Acta Otolaryngol 1985; 99:4-37.

5. Schiff M, Yoo TJ. Imunologic aspects of otologic disease: an overview. Laryngoscope 1985;95:259.

6. Stephens SDG, LuxonL, Hinchcliffe R. Immunological disorders and auditory lesions. Audiology 1982;21:128-148.

7. Mccabe BF. Autoimmune sensorioneural hearing loss. Ann Otol 1979;88:585-589.

8. Veldman JE, Roord JJ, O'Connor AF et al. Autoimmunity and inner ear disorders and immune complex mediated sensorioneural hearing loss. Laryngoscope 1984;94:501.

9. Veldman JE. Immune-mediated inner ear disorders. New syndromes and then etiopathogenesis. In: Veldman JE \& Mccabe BF. Oto-immunology. Amsterdam: Kugler Publ.; 1987. p.125-142.

10. Mccabe BF. Autoimmune inner ear disease: clinical varieties of presentation. in: Veldman JE \& Mccabe BF. Oto-immunology. Amsterdan: Kugler Pube; 1987. p.143-148.

11. Hughes GB, Kinney SE, Barna BP, Hamid MA, Calabrese LH. Autoimmune Vestibular dysfunction: preliminary report. Laryngoscope 1985;95:893-897.

12. Kemp P, Alexander L, Brown AM. Acoustic Emission cochleography: Practical aspects. Scand Audiol Suppl 1986;25:71-95.

13. Probst R, Lonsbury - Martin BL, Martini GK. A review of otoacustic Emissions. J Acoust Soc Am 1991;89(5):2027-2062.

14. Martin GK, Whitehead ML, Lonsbrery Martin BL. Potential of evoked otoacustic emissions for infant hearing screening. Sem Hear 1990;11:186-204.

15. Norton SJ \& Stover IJ. Otoacustic Emission. In: Katz J (ed). Handbook of clinical Audiology. $4^{\text {th }}$. ed. Baltimore: Williams \& Wilkins; 1994. p. 448-462.

16. Ferreira LA, Silveira JAM, Percebo DD, Gonçales F. Perda Auditiva Induzida por Ruído: Análise dos Achados de Audiometria Tonal, Potenciais Evocados do Tronco Cerebral e Emissões Otoacústicas Evocadas por Produto de Distorção. Revista Brasileira de Otorrinolaringologia 2001;67(1): 9-14.

17. Harris JP. Autoimmune or immune-mediated causes of deafness. West J Med 1991;154:719-20.

18. Bowman CA \& Nelson RA. Human leukocytic antigens in autoimmune sensorineural hearing loss; 1987:7-9.

19. Kanzaki J \& O-Uchi T. Circulating immune complexes in steroidresponsive sensorineural hearing loss and the long-term observation. Acta Otolaryngol 1983 (Suppl.) 393:77-84, D.T.; Bray.

20. Samara AD, Marques-Neto JF, Ermel MH, Maudonnet O. Audição na artrite reumatóide: recursos da impedanciometria e audiometria tonal. Rev Bras Reumat 1975;2:86-90.

21. Atra E, Pontes PAL, Goldenberg J, Nóbrega JL, Sato EI, Pollar DF. Estudo da audição no lúpus eritematoso disseminado. Rev Bras Reumat 1983;23(5):203-207. 